\title{
THz stimulated emission from simple superlattice in positive differential conductivity region
}

\author{
(C) A.A. Andronov ${ }^{1}$, A.V. Ikonnikov ${ }^{1}$, K.V. Maremianin ${ }^{1}$, V.I. Pozdnjakova ${ }^{1}$, Y.N. Nozdrin ${ }^{1}$, \\ A.A. Marmalyuk ${ }^{2}$, A.A. Padalitsa ${ }^{2}$, M.A. Ladugin'2, V.A. Belyakov ${ }^{3}$, \\ I.V. Ladenkov ${ }^{3}$, A.G. Fefelov ${ }^{3}$ \\ ${ }^{1}$ Institute for Physics of Microstructures RAS, Russian Academy of Sciences, \\ 603950 Nizhny Novgorod, Russia \\ 2 Sigm-Plus, \\ Moscow, Russia \\ ${ }^{3}$ Federal Government Unitary Enterprise „Salut", \\ Nizhny Novgorod, Russia \\ E-mail: andron@ipmras.ru
}

Narrow band emissions at $2.6-2.8 \mathrm{THz}$ are observed out of liquid helium cooled $1 \mathrm{~mm}$ disk chips prepared of a wafer with the very low $\mathrm{n}$ type doped weak barrier GaAs-GaAlAs superlattice of 1000 periods. The emissions are at about $8.0-18.0 \mathrm{~V}$ pulsed voltage applied to the chips in region of the chips positive DC differential conductivity that guaranties absence of inhomogeneous electric field domains in the chips. The emission frequency bands are estimated with a cyclotron resonance filter; the measurements show that the band width is of about that of the $\mathrm{THz}$ quantum cascade laser. By using long voltage pulses the chip heating above $100 \mathrm{~K}$ is achieved without substantial change in emission power. We speculate that the emission is super luminescence (amplification) of whispering gallery modes in the chips as a result of inverted Wannier-Stark level transitions under bias. The results are the first world demonstration of $\mathrm{THz}$ stimulated emission in a simple superlattice within region of positive $\mathrm{DC}$ differential conductivity; they give strong impetus for development of $\mathrm{THz}$ and higher frequency sources based on such simple superlattices; the sources should well compete with the $\mathrm{THz}$ quantum cascade lasers in particular at elevated temperatures.

\section{Acknowledgment}

The authors appreciate interest in our work by Andreas Wacker (Lund), support of work by V.I. Gavrilenko (IPM RAS), acknowledge contribution to this work by Alexander Andrianov and his group from Ioffe Institute, comments by S. Komiyama (Tokyo), and the SLs $X$-ray characterization by Yu.N. Drozdov and P.A. Yunin (IPM RAS).

The work was supported by Grants from RAS Programs and from RFBR Grant 15-02-05503-a. 